\title{
Determinantes de Risco de Liquidez em Cooperativas de Crédito: uma Abordagem a partir do Modelo Logit Multinomial
}

\section{Analysis of the Liquidity Risk in Credit Unions: a Logit Multinomial Approach}

\author{
Rosiane Maria Lima Gonçalves * \\ Doutoranda em Economia Aplicada pela UFV. \\ Professora Assistente da UFV/Campus Rio Paranaíba, Rio Paranaíba /MG, Brasil. \\ Marcelo José Braga \\ Pós-Doctor em Economia Agrícola pela Universidade da Califórnia, Estados Unidos. \\ Professor Adjunto do DER/UFV, Viçosa/MG, Brasil.
}

*Endereço: Universidade Federal de Viçosa - UFV/Campus Rio Paranaíba, Rodovia BR 354 - km 310, Rio Paranaíba/MG, 38810-000.E-mail: rosiane.goncalves@ufv.br 


\title{
ResUMo
}

O risco de liquidez nas instituições financeiras está associado ao desequilíbrio entre os ativos negociáveis e passivos exigíveis. Outros fatores também afetam a liquidez das cooperativas de crédito, como a maior utilização da cooperativa para empréstimos do que para depósitos e a incapacidade em promover a diversificação geográfica e de produtos. Nesse sentido, este estudo objetivou verificar, a partir de indicadores financeiros, qual é o risco de liquidez das cooperativas de economia e crédito mútuo de Minas Gerais e quais os determinantes desse risco. Foi utilizado o modelo de regressão logit multinomial, sendo as cooperativas classificadas em muito baixo, baixo, médio, alto e muito alto risco de liquidez. Os resultados analisados indicaram que valores menores dos indicadores utilização de capital de terceiros e provisionamento e valores maiores dos indicadores depósito total/operações de crédito e logaritmo do total de ativos tornam essas instituições mais líquidas.

Palavras-chave: cooperativas de economia e crédito mútuo; risco de liquidez; logit multinomial; Minas Gerais.

\begin{abstract}
Liquidity risk in financial institutions is associated to balance between working capital and financial demands. Other factors that affect credit union liquidity are an unanticipated increase of withdrawals without an offsetting amount of new deposits, and the lack of ability in promoting the product geographical diversification. The objective of this study is to analyze Minas Gerais state credit union liquidity risk and its factor determinants. Financial ratios and the multinomial logit model are used. The cooperatives were classified in five categories of liquidity risk: very low, low, medium, high and very high. The empirical results indicate that high levels of liquidity are related to smaller values of the outsourcing capital use, immobilization of the turnover capital, and provision ratios. So, they are associated to larger values of the deposit total/credit operations, and asset growth ratios.
\end{abstract}

Key words: credit unions; liquidity risk; logit multinomial; Minas Gerais. 


\section{INTRODUÇÃO}

As cooperativas de crédito são instituições financeiras que tornam o acesso ao crédito mais fácil e barato, visto que reduzem os juros bancários, especialmente as taxas de cheque especial e de empréstimos. Nessas instituições, o retorno excedente é distribuído aos sócios ou, por decisão de todos em Assembléia Geral, pode retornar, na forma de juros mais altos, sobre as aplicações (depósitos de longo prazo) ou na forma de menor custo, o que diminui as taxas de empréstimos e de prestação de serviços.

$\mathrm{O}$ risco de liquidez pode ser caracterizado pela insuficiência de recursos disponíveis para o cumprimento de obrigações. Em uma instituição financeira, está associado aos desequilíbrios entre ativos negociáveis e passivos exigíveis. As cooperativas de crédito têm sido afetadas pelo risco de liquidez, dada a incapacidade em promover a diversificação geográfica e de produtos, devido ao fato de todos os membros viverem na mesma área e de haver dificuldade de captação de recursos, os quais são obtidos, em maior parte, mediante depósitos realizados pelos sócios. No Estado de Minas Gerais, a gestão de risco das cooperativas de crédito é ainda incipiente, apesar de este ser o Estado brasileiro que apresenta o maior número de cooperativas de crédito. Assim, dada a ausência de estudos sobre risco de liquidez em cooperativas de crédito mineiras e dada a presença significativa destas no Estado, este estudo objetivou analisar qual é o risco de liquidez das cooperativas de economia e crédito mútuo de Minas Gerais e quais os condicionantes desse risco.

\section{Referencial Teórico}

Conforme Assaf (2003), o conceito de risco pode ser entendido de diversas maneiras e, nessa abrangência do entendimento, a avaliação de uma organização delimita-se aos componentes de seu risco total: econômico e financeiro. As principais causas do risco econômico são de natureza conjuntural, de mercado e do próprio planejamento e gestão da organização. O risco financeiro, de outro modo, está mais diretamente relacionado com o endividamento (passivos) da empresa e com sua capacidade de pagamento. Dessa maneira, pode-se deduzir que o risco total é definido pela sua parte sistemática (risco sistemático ou conjuntural) e não-sistemática (risco específico e próprio). 
O risco sistemático não tem como ser evitado, já que se relaciona com o ambiente externo da instituição, no qual as variáveis não podem ser controladas; mas devem, no entanto, ser conhecidas pelos administradores, para que a instituição esteja preparada para enfrentar seus efeitos. Já o risco não-sistemático ou próprio relaciona-se, diretamente, com o modelo de gestão da instituição, sendo inerente às decisões tomadas. Para uma instituição financeira, o nível de risco próprio dependerá, por exemplo, dos critérios adotados na concessão de empréstimos, do numerário mantido na conta disponibilidades para assegurar a liquidez e da diversificação da carteira de empréstimos.

Alguns tipos de risco foram caracterizados por Duarte (2003), que definiu risco de mercado como o que se refere aos retornos esperados por um investimento em decorrência de fatores como variação nas taxas de juros, nas taxas de câmbio, nos preços das ações, entre outros. Já o risco operacional é uma medida das possíveis perdas em uma instituição, caso seus sistemas, suas práticas e suas medidas de controle não resistam a falhas humanas ou situações adversas no mercado. O risco de crédito ocorre quando uma das partes de um contrato não consegue cumprir o pagamento de sua dívida, e o risco legal é uma medida das possíveis perdas em uma instituição, caso seus contratos não possam ser legalmente amparados por falta de representatividade de um negociador.

Conforme Palia e Porter (2003), o risco de liquidez decorre da inabilidade para satisfazer as exigências de caixa, quando necessário, podendo ser caracterizado como insuficiência de recursos disponíveis para o cumprimento das obrigações da instituição. Em uma instituição financeira pode ocorrer quando há ausência de dinheiro para atender à necessidade de saques dos clientes. O Artigo 2, da Resolução 2.804/2000 do Banco Central do Brasil [Bacen], definiu o risco de liquidez como a ocorrência de desequilíbrios entre ativos negociáveis e passivos exigíveis - descasamentos entre pagamentos e recebimentos - que possam afetar a capacidade de pagamento da instituição, levando-se em consideração as diferentes moedas e os prazos de liquidação de seus direitos e obrigações.

Para Saunders (2000), na gestão de passivos o banco recorre ao mercado interbancário de empréstimo em curto prazo. Todos os ajustes decorrentes da redução dos depósitos ocorrem no lado do passivo. De acordo com World Council of Credit Unions (2002), em muitos países as cooperativas de crédito não têm acesso a esses recursos, razão por que ficam sujeitas a severos riscos de liquidez. Os depósitos totais são freqüentemente usados para assegurar a existência de liquidez adequada, para satisfazer solicitações de saques dos sócios.

Madura (2003) apontou outros três motivos que tornam as cooperativas de crédito mais propensas ao risco de liquidez. Primeiramente, pode-se considerar 
que esse risco se torna especialmente maior quando os membros da cooperativa são empregados de uma única empresa. Neste caso, se a empresa demitir vários trabalhadores, muitos sócios podem, simultaneamente, sofrer problemas financeiros e promover a retirada de seus depósitos ou podem tornar-se inadimplentes com relação aos empréstimos tomados. Com isso, a cooperativa poderia tornar-se ilíquida. O segundo motivo está relacionado com a incapacidade dessas cooperativas em promover a diversificação geográfica e de produtos. Isso decorre do fato de todos os membros viverem na mesma área. Como último motivo para o risco de liquidez ser mais freqüente nas cooperativas de crédito do que nas demais instituições financeiras, têm-se que, em uma situação de necessidade de recursos, as cooperativas enfrentam maiores dificuldades de captação que os bancos, pois seus recursos são obtidos, em maior parte, dos depósitos realizados pelos sócios.

Segundo Bank for International Settlements (2004), a liquidez é crucial à viabilidade contínua de qualquer instituição financeira, e cada instituição deve ter sistemas adequados para medir, monitorar e controlar o risco de liquidez e deveriam também avaliar a suficiência de capital, dado o seu próprio perfil de liquidez, e a liquidez dos mercados em que operam.

A preocupação com as situações de risco, enfrentadas pelas instituições financeiras na década de 70, resultou no surgimento do Comitê de Basiléia de Supervisão Bancária, formado por representantes dos países do G-10, isto é, Alemanha, Bélgica, Canadá, Estados Unidos, França, Holanda, Itália, Japão, Reino Unido e Suécia. Esse comitê formalizou alguns princípios para gestão do risco de liquidez, com a publicação do documento Sound Practices for Managing Liquidity, em fevereiro de 2000, ao propor que bancos comerciais internacionais administrem a liquidez em uma base global de técnicas e práticas vitais para a continuidade de suas atividades.

O Bacen, em consonância com os princípios do gerenciamento de liquidez do Comitê de Basiléia, estabeleceu, por meio da Resolução 2.804 de dezembro de 2000, que as instituições financeiras devem manter sistemas de controle do risco de liquidez estruturados, de acordo com seus perfis operacionais e periodicamente reavaliados, com vistas no acompanhamento permanente das posições assumidas em todas as operações praticadas nos mercados financeiros e de capitais, de forma a evidenciar o risco de liquidez decorrente das atividades por elas desenvolvidas. Assim, de acordo com a resolução, as instituições devem, entre outras atribuições:

1. Manter, de forma adequadamente documentada, os critérios e a estrutura estabelecidos para o controle do risco de liquidez. 
2. Realizar avaliações voltadas à identificação de mecanismos e instrumentos que permitam a obtenção dos recursos necessários à reversão de posições que coloquem em risco a situação econômico-financeira da instituição, englobando as alternativas de liquidez disponíveis nos mercados financeiros e de capitais.

3. Elaborar análises econômico-financeiras que permitam avaliar o impacto dos diferentes cenários na condição de liquidez de seus fluxos de caixa, levando em consideração, até mesmo fatores internos e externos à instituição.

A Central das Cooperativas de Economia e Crédito do Estado de Minas Gerais Ltda. [CECREMGE] também se tem comprometido com a gestão de riscos, com vistas em atender às exigências do Bacen, mediante normas internas que dispõem sobre o controle do risco de liquidez por meio de limites estipulados para a concessão de empréstimos e constituição da reserva compulsória de liquidez em relação à captação mensal das cooperativas de crédito. Estes valores são remunerados em 100\% do Certificado de Depósitos Interfinanceiros [CDI] sobre depósitos, diferentemente do compulsório recolhido pelo Bacen junto aos bancos comerciais, o qual fica congelado pelo Banco Central.

Para que o risco de liquidez possa ser avaliado, gerenciado e controlado, é necessário que sejam conhecidos os seus principais determinantes, ou seja, a partir da estrutura das contas ativas e passivas e suas inter-relações, identificar os principais fatores que têm maior influência na condição de risco da instituição.

Conforme a Comissão de Gestão de Riscos da Federação Brasileira de Bancos (2005), cujo objetivo é fornecer subsídios ao processo de gestão do risco de liquidez, podem-se utilizar indicadores provenientes das relações das contas ativas e passivas dos balanços financeiros na avaliação da situação de liquidez da instituição, os quais são úteis por permitirem análises comparativas entre períodos diferentes ou em relação a outras instituições com o mesmo perfil.

Diversos autores utilizaram indicadores financeiros na análise de instituições financeiras. Aspachs, Nier e Tiesset (2005), na análise dos determinantes das políticas de liquidez de bancos no Reino Unido, utilizaram, como variável dependente em suas regressões, a relação entre recursos líquidos e depósitos totais, visando determinar a liquidez inerente ao balancete da instituição. Como variáveis explicativas, foram utilizados indicadores de lucratividade, crescimento dos empréstimos e logaritmo do total de ativos.

Durán, Matarrita e Bronn (2004) apresentaram, em seu estudo, o caso da Argentina, na qual a qualificação de risco por entidades especializadas é aplicada pelo Banco Central da República Argentina [BCRA] a todas as entidades 
financeiras, mesmo às cooperativas de crédito. Os indicadores de rentabilidade, endividamento, capitalização, liquidez e provisionamento são considerados aspectos mínimos que devem ser analisados pelas qualificadoras. O Banco de Portugal (2004), em seu Relatório de Estabilidade Financeira, também abordou o acompanhamento do risco de liquidez feito pelo seu sistema bancário, no qual os principais indicadores utilizados na análise do risco de liquidez são baseados em informações contábeis.

Conforme Richardson (2002), desde 1990, o Conselho Mundial de Cooperativas de Crédito vem utilizando um sistema de monitoramento que inclui relações entre as contas passivas e ativas das demonstrações financeiras, conhecido como PEALS - proteção, estrutura financeira efetiva, qualidade de ativos, liquidez e sinais de crescimento - em que cada palavra representa áreas-chaves das operações das cooperativas de crédito.

Matias e Siqueira (1996) e Bressan (2002), ao estudarem os determinantes da insolvência em instituições financeiras, usaram as relações contábeis destas instituições, considerando indicadores de estrutura, solvência, custos e despesas, rentabilidade e crescimento.

\section{Metodologia}

\section{Base de Dados}

Os dados utilizados são provenientes de balanços patrimoniais mensais de 69 cooperativas de economia e crédito mútuo do Estado de Minas Gerais, no período de fevereiro de 2003 a maio de 2005, num total de 28 balanços. Essas informações foram de natureza secundária e foram obtidas da Cecremge.

\section{Determinantes do Risco de Liquidez e a Aplicação do Modelo Multinomial}

O estudo baseou-se em cooperativas de economia e crédito mútuo mineiras, que são classificadas em diferentes faixas de risco de liquidez. A variável dependente foi definida, conforme estudo realizado por Aspachs et al. (2005), pela análise dos determinantes das políticas de liquidez de bancos no Reino Unido, sendo a razão entre recursos líquidos das cooperativas de crédito (soma das disponibilidades, aplicações interfinanceiras de liquidez, títulos e valores mobiliários e relações interfinanceiras) e depósitos totais denominada Reserva de Liquidez. 
De acordo com Greene (2003), os modelos probit e logit podem ser considerados, quando a variável dependente é composta por diferentes categorias. A utilização do modelo probit tem sido limitada pelo fato de apresentar maior complexidade matemática. Já o modelo logit é extensamente usado em diferentes campos de pesquisa, até mesmo em economia, pesquisa de mercado e engenharia. Segundo Maddala (1986), o modelo probit só pode ser aplicado para pequeno número de alternativas, três ou quatro, pelo fato de os cálculos envolverem estimação de múltiplas integrais. Kaplan e Urwitz (1979) compararam os modelos estimados por máxima verossimilhança, como o modelo logit, e a análise discriminante e consideraram como a principal vantagem do primeiro modelo em relação ao segundo o fato de, sob condições gerais, os estimadores de máxima verossimilhança possuírem estimadores consistentes e serem assintoticamente eficientes.

O modelo logit multinomial foi usado, por Theil (1969), Cragg e Uhler (1970) e Schimit e Strauss (1975), nos seguintes estudos: escolhas das formas de transporte, demanda de automóveis e determinantes da ocupação profissional, respectivamente. Kaplan e Urwitz (1979) aplicaram esse método no estudo de ratings de crédito, considerando a importante contribuição para análise de risco de crédito. Podem ser citados também estudos atuais, como o de Lawrence e Arshadi (1995), que analisaram as formas de resoluções dos problemas ocorridos com empréstimos concedidos por bancos comerciais, como execução de hipoteca, liquidações e renegociação e de Young (2003), que estudou a fragilidade financeira de novos bancos nos Estados Unidos, no período de 1980 a 1985, e considerou a variável dependente com quatro categorias: bancos que sobreviveram, bancos que faliram, aquisições bancárias, ou bancos convertidos em filiais.

Considere j como um índice que representa a variável de resposta com diferentes categorias, estando associada a cada categoria diferentes probabilidades. Tendo a variável de resposta j categorias, devem-se estimar j-1 equações, sendo uma das categorias considerada como categoria de base.

Com base na análise de probabilidades, a estrutura geral deste modelo pode ser apresentada, conforme Greene (2003), por

$$
P_{i}=\operatorname{Pr} o b\left(Y_{i}=j\right)=\frac{e^{\left(\beta_{j}^{\prime} x_{i}\right)}}{\sum_{k=0}^{J} e^{\left(\beta_{k}^{\prime} x_{i}\right)}}, \operatorname{para} \mathrm{j}=0,1,2,3,4
$$

em que $\mathrm{P}_{\mathrm{i}}$ representa as diferentes probabilidades de risco de liquidez: $\mathrm{Y}_{\mathrm{i}}=0$, se a cooperativa está com muito baixo risco de liquidez; $\mathrm{Y}_{\mathrm{i}}=1$, baixo risco de liquidez; $\mathrm{Y}_{\mathrm{i}}=2$, médio risco de liquidez; $\mathrm{Y}_{\mathrm{i}}=3$, alto risco de liquidez; e $\mathrm{Y}_{\mathrm{i}}=4$, muito alto 
risco de liquidez; $x_{i}$, vetor de variáveis explicativas; $\beta$, vetor de parâmetros a serem estimados e e base dos logaritmos naturais.

As diferentes faixas de risco de liquidez foram definidas com base no sistema de monitoramento da Cecremge que utiliza uma matriz de riscos, composta por diversos indicadores, definida com base em critérios utilizados pelo Bacen, Sistema de Cooperativas de Crédito do Brasil [SICOOB] e mercado financeiro. Para os indicadores que não possuem legislação específica, a Cecremge criou faixas de risco de acordo com o mercado e experiência própria em auditorias realizadas junto às cooperativas filiadas. No caso do indicador de risco de liquidez, quanto mais próximo de um, menor será o risco, conforme Tabela 1.

\section{Tabela 1: Faixas e Categorias de Risco de Liquidez}

\begin{tabular}{ccc}
\hline Faixas & Categorias & Risco de liquidez \\
\hline$\geq 55,00$ & 0 & Muito baixo risco \\
$45,00-54,99$ & 1 & Baixo risco \\
$35,00-44,99$ & 2 & Médio risco \\
$20,00-34,99$ & 3 & Alto risco \\
$0,00-19,99$ & 4 & Muito alto risco \\
\hline
\end{tabular}

Fonte: resultados da pesquisa.

A equação 1 é interagida de tal forma, que um conjunto de parâmetros é calculado para cada categoria considerada, fornecendo as equações estimadas um conjunto de probabilidades para $\mathrm{J}+1$ categorias de resposta, com $\mathrm{x}_{\mathrm{i}}$ variáveis explicativas. Devese, no entanto, remover a indeterminação do modelo. Uma normalização que resolve o problema é $\beta_{0}=0$, a qual se faz necessária pelo fato de a soma das probabilidades ser igual a 1 , sendo necessários $\mathrm{J}$ vetores de coeficientes para determinar as $\mathrm{J}+1$ probabilidades. Assim, as probabilidades podem ser definidas por

$$
P_{i}=\operatorname{Pr} o b\left(Y_{i}=j\right)=\frac{e^{\left(\beta^{\prime}{ }_{j} x_{i}\right)}}{1+\sum_{k=1}^{J} e^{\left(\beta^{\prime}{ }_{k} x_{i}\right)}} \operatorname{para} \mathrm{j}=0,2, \ldots, \mathrm{J}, \beta_{0}=0 .
$$

O modelo pode ser reescrito em termos do logaritmo da razão das probabilidades:

$$
\ln \frac{P_{i j}}{P_{i k}}=x_{i}^{\prime}\left(\beta_{j}-\beta_{k}\right)=x_{i}^{\prime} \beta_{j} \text { se } \mathrm{k}=0
$$

Os betas indicam como as variáveis explicativas afetam o logaritmo da razão entre a probabilidade do risco de liquidez, do tipo j, e a probabilidade do risco de liquidez, do tipo $\mathrm{k}$. 
Como as probabilidades não são conhecidas, torna-se necessário fazer a estimação por máxima verossimilhança, considerando, para cada indivíduo, $\mathrm{d}_{\mathrm{ij}}=1$, se a alternativa $\mathrm{j}$ for escolhida pelo individuo $\mathrm{i}$, e 0 se não, para $\mathrm{J}-1$ possíveis resultados. Assim, para cada i, um e somente um dos $\mathrm{d}_{\mathrm{ij}}$ 's será 1 (Greene, 2003).

$$
L n L=\sum_{i=1}^{n} \sum_{j=0}^{j} d_{i j} \ln \operatorname{Pr} o b\left(Y_{i}=j\right) .
$$

Assim, os parâmetros do modelo são obtidos por

$$
\frac{\partial \ln L}{\partial \beta_{j}}=\sum_{i}\left(d_{i j}-P_{i j}\right) x_{i} \text { para } \mathrm{j}=1, \ldots, \mathrm{J}
$$

Ao diferenciar a equação 1, encontra-se o efeito marginal das variáveis explicativas sobre as diferentes probabilidades de risco de liquidez das cooperativas de economia e crédito mútuo de Minas Gerais.

$$
\frac{\partial \mathrm{P}_{\mathrm{i}}}{\partial x_{i}}=\mathrm{P}_{\mathrm{j}}\left[\beta_{j}-\sum_{k=0}^{J} P_{k} \beta_{k}\right]=\mathrm{P}_{\mathrm{j}}\left[\beta_{j}-\bar{\beta}\right] .
$$

Greene (2003) enfatizou que os sinais dos coeficientes estimados e dos efeitos marginais, não necessariamente, serão os mesmos.

Neste estudo, segue a apresentação da forma estrutural do modelo:

$$
\begin{aligned}
& \ln \left(P_{1} / P_{0}\right)=\beta_{0}+\beta_{1} C A P_{i}+\beta_{2} A L_{i}+\beta_{3} \operatorname{Im} o b C G_{i}+\beta_{4} \log A T_{i}+\beta_{5} D T A T_{i}+ \\
& +\beta_{6} E_{i}+\beta_{7} U C T_{i}+\beta_{8} E N C_{i}+\beta_{9} C V_{i}+\beta_{10} A D x D V_{i}+\beta_{11} \operatorname{Pr}_{i}+\beta_{12} V L_{i}+ \\
& +\beta_{13} C_{i}+\beta_{14} \text { DTOC }_{i}+\beta_{15} \text { TOT }_{i}+\beta_{16} \text { RPL }_{i}+\beta_{17} \mathrm{CrApl}_{i}+\beta_{18} \mathrm{CrCap}_{i}+ \\
& +\beta_{19} C r A T_{i}+\varepsilon_{i} \\
& \ln \left(P_{2} / P_{0}\right)=\beta_{20}+\beta_{21} C A P_{i}+\beta_{22} A L_{i}+\beta_{23} \operatorname{Im} o b C G_{i}+\beta_{24} \log A T_{i}+\beta_{25} D T A T_{i}+ \\
& +\beta_{26} E_{i}+\beta_{27} U C T_{i}+\beta_{28} E N C_{i}+\beta_{29} C V_{i}+\beta_{30} A D x D V_{i}+\beta_{31} \operatorname{Pr}_{i}+\beta_{32} V L_{i}+ \\
& +\beta_{33} C_{i}+\beta_{34} D_{T O C}+\beta_{35} \mathrm{TOT}_{i}+\beta_{36} \mathrm{RPL}_{i}+\beta_{37} \mathrm{CrApl}_{i}+\beta_{38} \mathrm{CrCap}_{i}+ \\
& +\beta_{39} C r A T_{i}+\varepsilon_{i}
\end{aligned}
$$




$$
\begin{aligned}
\ln \left(P_{4} / P_{0}\right) & =\beta_{60}+\beta_{61} C A P_{i}+\beta_{62} A L_{i}+\beta_{63} \operatorname{Im} o b C G_{i}+\beta_{64} \log A T_{i}+\beta_{65} D T A T_{i}+ \\
& +\beta_{66} E_{i}+\beta_{67} U C T_{i}+\beta_{68} E N C_{i}+\beta_{69} C V_{i}+\beta_{70} A D x D V_{i}+\beta_{71} \operatorname{Pr}_{i}+\beta_{72} V L_{i}+ \\
& +\beta_{73} C_{i}+\beta_{74} D T O C_{i}+\beta_{75} T O T_{i}+\beta_{76} R P L_{i}+\beta_{77} C r A p l_{i}+\beta_{78} C r C a p_{i}+ \\
& +\beta_{79} C r A T_{i}+\varepsilon_{i}
\end{aligned}
$$

em que

ln é logaritmo natural;

$\mathrm{P}_{\mathrm{i}}$, probabilidade de risco de liquidez das cooperativas de crédito;

$\beta_{\mathrm{i}}(\mathrm{i}=0, \ldots, 19)$, parâmetros a serem estimados;

$\mathrm{CAP}_{\mathrm{i}}$, Capitalização = Patrimônio Líquido/ Passivo Real ${ }^{(1)}$;

$\mathrm{AL}_{\mathrm{i}}$, Alavancagem = Captação Total/Patrimônio Líquido;

ImobCG, Imobilização do Capital em Giro = Capital Imobilização /(Passivo Circulante + Passivo Exigível a Longo Prazo + Patrimônio Líquido);

$\log \mathrm{AT}_{\mathrm{i}}$, Logaritmo do Ativo Total;

DTAT $_{\text {i }}$, razão entre Depósitos Totais e Ativos Totais;

$\mathrm{E}_{\mathrm{i}}$, Endividamento = $($ Passivo Circulante + Passivo Exigível a Longo Prazo $) /$ (Patrimônio Líquido);

$\mathrm{UCT}_{\mathrm{i}}$, Utilização do Capital de Terceiros $=($ Passivo Circulante e Passivo Exigível a Longo Prazo)/Capital Total (Passivo Circulante, Passivo Exigível a Longo Prazo e Patrimônio Líquido);

$\mathrm{ENC}_{\mathrm{i}}$, Encaixe $=$ Disponibilidades $/$ Depósitos à Vista;

$\mathrm{CV}_{\mathrm{i}}$, Cobertura Voluntária $=$ Disponibilidades $/$ Passivo Real;

$\mathrm{AD} / \mathrm{DV}_{\mathrm{i}}$, razão entre Adiantamento a Depositante e Depósito à Vista;

$\operatorname{Pr}_{\mathrm{i}}$, Provisionamento = Provisões de Crédito para Liquidação Duvidosa/ Operação de Crédito;

$\mathrm{VL}_{\mathrm{i}}$, Volume de Crédito = Operações de Crédito/Patrimônio Líquido;

$\mathrm{C}_{\mathrm{i}}$, Comprometimento = Provisões de Crédito para Liquidação Duvidosa/ Patrimônio Líquido;

DTOC $_{\mathrm{i}}$, razão entre Depósitos Totais e Operações de Crédito; 
$\mathrm{TOT}_{\mathrm{i}}$, Custo e Despesa $=$ Despesa Total/Captação Total ${ }^{(2)}$;

$\mathrm{RPL}_{\mathrm{i}}$, Rentabilidade do Patrimônio Líquido = Resultado Líquido/Patrimônio Líquido;

$\mathrm{CrApl}_{\mathrm{i}}$, Crescimento das Aplicações Totais $=$ Aplicação Total ${ }^{(3)}$ do Último Período/Aplicação Total do Período Anterior;

CrCap ${ }_{i}$, Crescimento das Captações Totais = Captação Total do Último Período/ Captação Total do Período Anterior;

$\mathrm{CrAT}_{\mathrm{i}}$, Crescimento do Ativo Total $=$ Ativo Total do Último Período $/$ Ativo Total do Período Anterior; e

$\varepsilon_{\mathrm{i}}$, termo de erro aleatório.

No modelo analisado foi considerada como categoria de base a probabilidade de as cooperativas estarem com muito baixo risco de liquidez. A probabilidade de ocorrência de cada resposta, de acordo com este modelo, varia entre as cooperativas de crédito mútuo de acordo com os diferentes valores dos indicadores considerados.

Para identificar se as variáveis explicativas inseridas no modelo exerciam algum tipo de influência sobre a variável dependente, foi realizada a análise da razão estatística de máxima verossimilhança (LR statistic). O LR statistic é igual a -2 (FVLI - FVLR), em que FVLI é função verossimilhança em log irrestrita (FVLI), obtida pela regressão formada pelos coeficientes e intercepto, como a equação 7 do modelo de regressão; FVLR, função verossimilhança em $\log$ com restrição (FVLR), em que se consideram todos os coeficientes de inclinação iguais a zero, mantendo somente o intercepto. Se a restrição for válida, então FVLI = FVLR, e o modelo não terá significância global.

No desenvolvimento do modelo, para teste dos indicadores, foi utilizado o método de regressão stepwise que, segundo Draper e Smith (1966), é um método que procura identificar a contribuição dada por cada variável, sendo retiradas do modelo as que não fossem significativas. Este processo perdura até que variáveis não possam ser admitidas ou rejeitadas, havendo um reexame, em todas as fases da regressão, das variáveis incorporadas no modelo em fases anteriores. Esse método visou encontrar variáveis que fossem significativas para todas as faixas de risco, dado que, no modelo multinomial, os coeficientes, os sinais e o nível de significância mudam entre as categorias analisadas.

Foi também aplicado um teste para verificar a propriedade da independência das alternativas irrelevantes. De acordo com Greene (2003), no modelo logit 
multinomial, as razões das probabilidades são independentes das demais alternativas. A propriedade do modelo logit, por meio do qual $\frac{P_{i j}}{P_{i k}}$ é independente das probabilidades restantes, é chamada independência das alternativas irrelevantes. Se uma das alternativas for verdadeiramente irrelevante e for omitida do modelo, não haverá mudanças nos coeficientes estimados.

O teste da independência das alternativas irrelevantes considera que C, de acordo com Maddala (1986), seja um conjunto completo de alternativas; D, um subconjunto de $\mathrm{C} ; \hat{\beta}_{C}$, o coeficiente obtido pelo modelo logit multinomial com todas as alternativas; e $\hat{\beta}_{D}$ o coeficiente correspondente ao modelo restrito estimado, D. Na hipótese nula, em que a propriedade da independência das alternativas irrelevantes seja assegurada, $\hat{\beta}_{D}-\hat{\beta}_{C}$ será zero. Se esta propriedade falhar, o resultado será diferente de zero.

Para a realização do teste, o modelo de regressão foi estimado cinco vezes: uma com todas as faixas de risco de liquidez e outras com subconjuntos de alternativas, baseado na exclusão de alguma opção.

\section{Resultados e Discussões}

\section{Logit Multinomial e Determinantes do Risco de Liquidez}

Para classificar as cooperativas nas diferentes faixas de risco foi utilizado o indicador reserva de liquidez, sendo as faixas de risco definidas com base no sistema de monitoramento realizado pela Cecremge.

Pode-se verificar, na Tabela 2, que o maior número de observações se concentra na classificação de muito baixo risco de liquidez, o que indica que boa parte das cooperativas analisadas se tem preocupado com seus níveis de risco de liquidez, buscando manter níveis mais baixos, para assegurar a confiabilidade dos que depositam na instituição. 
Tabela 2: Classificação das Cooperativas de Economia e Crédito Mútuo de Minas Gerais, com base nas Diferentes Faixas de Risco de Liquidez, no Período de Fevereiro de 2003 a Maio de 2005

\begin{tabular}{ccc}
\hline Faixas de risco de liquidez & Número de observações & Percentual \\
\hline Muito baixo risco & 713 & $36,90 \%$ \\
Baixo risco & 325 & $16,82 \%$ \\
Médio risco & 294 & $15,22 \%$ \\
Alto risco & 337 & $17,44 \%$ \\
Muito alto risco & 263 & $13,61 \%$ \\
\hline Total & $1.932^{(4)}$ & $100 \%$ \\
\hline
\end{tabular}

Fonte: resultados da pesquisa.

Foi analisada a razão estatística de máxima verossimilhança (LR statistic) de cada indicador, para determinar os que tinham maior influência nas diferentes faixas de risco de liquidez e verificada a correlação entre esses indicadores. Foi excluído o indicador correlacionado que apresentasse o menor LR statistic, ou seja, que tinha menor influência na variável dependente.

Identificadas as variáveis que apresentaram baixa correlação e alto $L R$ statistic, foi aplicado o método stepwise, com vistas em identificar a contribuição de variável para o modelo de regressão e encontrar o melhor modelo ajustado.

O modelo resultante da regressão logit multinomial, pelo método stepwise, selecionou, entre os 19 indicadores avaliados, apenas 4 como determinantes do risco de liquidez, nas diferentes faixas de risco consideradas. A Tabela 3 apresenta as estatísticas dessas variáveis, sendo elas: utilização do capital de terceiros, depósito total/operação de crédito, logaritmo do total de ativos e provisionamento.

Tabela 3: Estatística Descritiva das Variáveis Presentes no Modelo de Regressão, no Período de Fevereiro de 2003 a Maio de 2005

\begin{tabular}{ccccc}
\hline & Uct & Dpt/OpCr & LogAt & $\mathrm{P}$ \\
\hline & & & & \\
Média & 0,73 & 1,31 & 6,60 & 0,07 \\
Desvio padrão & 0,19 & 1,29 & 0,47 & 0,16 \\
Mínimo & 0,14 & 0,04 & 5,53 & 0,00 \\
Máximo & 1,54 & 15,74 & 8,17 & 2,15 \\
\hline
\end{tabular}

Fonte: resultados da pesquisa.

Uct = Utilização do capital de terceiros; Dpt/OpCr = Depósito Total/Operação de Crédito; LogAt $=$ Logaritmo do Total de Ativos; e P = Provisionamento. 
Os resultados encontrados na Tabela 3 indicam que, em média, as cooperativas de economia e crédito mútuo analisadas, utilizam 73\% do capital de terceiros em relação ao capital total existente na instituição. A variável que apresentou maior dispersão dos dados em torno da média foi a razão entre depósito total e operação de crédito. Em média, as cooperativas captam mais depósitos do que concedem aos cooperados em forma de crédito, relação favorável a saúde financeira da instituição. Considerando o valor mínimo encontrado para esse indicador, verificase que algumas cooperativas enfrentam dificuldades na captação de recursos, visto que o valor mínimo de 0,04 indica um desajuste entre captação e crédito fornecido.

Verifica-se que, em média, não há muita variabilidade entre os valores máximo e mínimo encontrados para logaritmo do total de ativos, variável utilizada para representar o tamanho da instituição, o que indica certa semelhança no tamanho das instituições. Com relação ao provisionamento, observa-se, pelos valores máximo e mínimo, duas realidades amplamente distintas entre as instituições analisadas. O valor mínimo indica que há cooperativas que não fazem nenhuma provisão para o crédito de liquidação duvidosa, enquanto o valor máximo reflete alta inadimplência esperada por algumas cooperativas.

O modelo de regressão logit multinomial pode ser considerado globalmente válido, pela análise da razão estatística de máxima verossimilhança ( $L R$ statistic) apresentada nas notas da Tabela 4, sendo o p-valor do teste de validade global do modelo, dado pela Probabilidade (LR statistic), altamente significativo. O $R^{2}$ McFadden encontrado indica que o modelo teve bom grau de ajustamento, o qual foi igual a 0,30 .

Foi verificada a propriedade da independência das alternativas irrelevantes, a qual também assegura a validade do modelo logit multinomial. Com base em Greene (2003), pode-se afirmar que o modelo não possui alternativas irrelevantes, ou seja, todas as faixas de risco consideradas são importantes, já que, ao omitir uma a uma no modelo de regressão, houve mudança nos coeficientes estimados. 
Tabela 4: Resultados do Modelo de Regressão Logit Multinomial da Probabilidade de Risco de Liquidez das Cooperativas de Economia e Crédito Mútuo de Minas Gerais, no Período de Fevereiro de 2003 a Maio de 2005

\begin{tabular}{rccccc}
\hline & Constante & Uct & Dpt/OpCr & LogAt & P \\
\hline $\ln \frac{P_{1}}{P_{0}}$ & $-2,10$ & 15,31 & $-3,76$ & $-0,75$ & 5,56 \\
$\ln \frac{P_{2}}{P_{0}}$ & $(0,09)^{* * *}$ & $(0,00)^{*}$ & $(0,00)^{*}$ & $(0,00)^{*}$ & $(0,00)^{*}$ \\
$\ln \frac{P_{3}}{P_{0}}$ & $-3,56$ & 23,54 & $-7,55$ & $-0,85$ & 9,85 \\
$\ln \frac{P_{4}}{P_{0}}$ & $(0,01)^{*}$ & $(0,00)^{*}$ & $(0,00)^{*}$ & $(0,00)^{*}$ & $(0,00)^{*}$ \\
\hline
\end{tabular}

Fonte: resultados da pesquisa.

Os valores entre parênteses são os P-valores.

*significativo a $1 \%$; ** significativo a $5 \%$; *** significativo a $10 \%$.

Os valores subscritos em P representam cada faixa de risco de liquidez: $0=$ muito baixo risco de liquidez; 1 = baixo risco de liquidez; 2 = médio risco de liquidez; 3 = alto risco de liquidez; e 4 = muito alto risco de liquidez; $L R$ statistic $=1651,14$.

Probabilidade $($ LR statistic $)=0,0000$.

$R^{2}$ McFadden $=0,30$.

Os coeficientes estimados, apresentados na Tabela 4, dão a variação no logaritmo da razão entre as diferentes probabilidades de risco de liquidez e a categoria de base $\left(P_{\mathrm{o}}\right)$, dada a mudança unitária nas variáveis consideradas. Para se obter a influência direta de cada variável explicativa nas probabilidades de risco de liquidez das cooperativas de economia e crédito mútuo analisadas, foram calculados os efeitos marginais, apresentados na Tabela 5. 
Tabela 5: Efeitos Marginais das Variáveis Explicativas do Modelo Multinomial de Regressão da Probabilidade de as Cooperativas de Economia e Crédito Mútuo de Minas Gerais Estarem em Risco de Liquidez, no Período de Fevereiro de 2003 a Maio de 2005

\begin{tabular}{ccccc}
\hline $\begin{array}{c}\text { Faixas de risco } \\
\text { de liquidez }\end{array}$ & Uct & Dpt/OpCr & LogAt & P \\
\hline Muito baixo risco & $-4,96$ & 1,45 & 0,21 & $-1,95$ \\
& $(0,00)^{*}$ & $(0,00)^{*}$ & $(0,00)^{*}$ & $(0,00)^{*}$ \\
Baixo risco & 1,48 & $-0,22$ & $-0,09$ & 0,44 \\
& $(0,00)^{*}$ & $(0,00)^{*}$ & $(0,00)^{*}$ & $(0,09)^{* *}$ \\
Médio risco & 1,68 & $-0,58$ & $-0,05$ & 0,74 \\
& $(0,00)^{*}$ & $(0,00)^{*}$ & $(0,01)^{*}$ & $(0,00)^{*}$ \\
Alto risco & 1,42 & $-0,50$ & $-0,05$ & 0,65 \\
& $(0,00)^{*}$ & $(0,00)^{*}$ & $(0,00)^{*}$ & $(0,00)^{*}$ \\
Muito alto risco & 0,38 & $-0,15$ & $-0,01$ & 0,12 \\
& $(0,00)^{*}$ & $(0,00)^{*}$ & $(0,00)^{*}$ & $(0,00)^{*}$ \\
\hline
\end{tabular}

Fonte: resultados da pesquisa.

Os valores entre parênteses são os P-valores.

*significativo a $1 \%$; ** significativo a $10 \%$.

Muito baixo risco de liquidez $=\operatorname{Prob}[\mathrm{Y}=0]$; baixo risco de liquidez $=\operatorname{Prob}[\mathrm{Y}=1]$; médio risco de liquidez $=\operatorname{Prob}[Y=2]$; alto risco de liquidez $=\operatorname{Prob}[\mathrm{Y}=3]$; e muito alto risco de liquidez = Prob[Y $=4]$.

O indicador utilização de capital de terceiros identifica qual a proporção do capital de terceiros em relação ao capital total da cooperativa. Na Tabela 5, verifica-se que esse indicador apresentou sinal negativo, para a probabilidade de muito baixo risco de liquidez, e sinal positivo, para as demais probabilidades. O aumento de $\mathrm{R} \$ 1,00$ na utilização de capital de terceiros, mantidas as demais variáveis constantes, reduziu a probabilidade de as cooperativas de economia e crédito mútuo mineiras estarem com muito baixo risco de liquidez em 4,96 e aumentou a probabilidade de alto risco de liquidez em 1,42, no período considerado.

Esse foi o indicador de maior impacto nas diferentes probabilidades de risco de liquidez, o que pode, em grande parte, ser explicado pelo fato de a maior utilização de capital de terceiros pelas cooperativas representar maiores riscos, já que essas instituições, normalmente, têm dificuldades de aumentar o volume de capital próprio. Essa dificuldade de capitalização deriva, principalmente, de dois fatores: primeiro, normalmente, o sócio não assume a cooperativa como empreendimento próprio; segundo, as quotas partes integralizadas não são transacionáveis no mercado financeiro. Assim, o sócio não tem expectativa de retorno pelo investimento realizado.

O indicador de provisionamento mostra o quanto dos créditos concedidos pela cooperativa é de liquidação duvidosa, ou seja, mostra qual o percentual de capital concedido está em atraso ou inadimplente. Quanto maior o provisionamento, maior será o risco, o que indica que a instituição tem realizado operações de baixa liquidez. 
Pelos resultados, tudo o mais constante, constata-se que o impacto marginal do provisionamento nas faixas muito baixo e médio risco de liquidez foi mais significativo, sendo a probabilidade marginal de $-1,95$ e $+0,74$, respectivamente. Esse resultado reflete que provisionamento e risco de liquidez variaram no mesmo sentido, ou seja, aumentos no montante de capital provisionado contribuíram para retirar as cooperativas de economia e crédito mútuo analisadas de uma situação de muito baixo risco de liquidez, situando-as em faixas mais elevadas de risco, no período de fevereiro de 2003 a maio de 2005 (Tabela 5).

O indicador, dado pela razão entre depósito total e operações de crédito, apresenta a relação entre as duas contas, que devem estar em equilíbrio na instituição para assegurar sua liquidez. Se a cooperativa fornecesse mais crédito do que capta em forma de depósito, estará reduzindo sua liquidez para atender às demandas de capital mais imediatas, como o resgate de depósitos à vista, o que comprometeria sua capacidade de saldar compromissos. Entretanto, é importante ressaltar que depósito total inferior às operações de crédito será aceitável, quando a cooperativa possuir boa capitalização, ou seja, quando o seu patrimônio líquido tiver capacidade para atender às demandas de capital, caso necessário. Dessa forma, a cooperativa reduz a necessidade de financiamento externo, não se comprometendo com dívidas que são onerosas para a instituição.

Os resultados mostraram que, mantidas as demais variáveis constantes, o aumento de $\mathrm{R}$ \$1,00 na razão entre depósito total e operações de crédito contribuiu para aumentar a probabilidade de as cooperativas de economia e crédito mútuo do Estado de Minas Gerais estarem com muito baixo risco em 1,45 e reduziu em 0,50 a probabilidade de alto risco, no período analisado.

Verifica-se que o equilíbrio entre o que a cooperativa capta e o que ela empresta é um importante determinante do grau de risco de liquidez. Esse resultado pode ser mais bem explicado por Madura (2003), que considerou que o crescimento dos empréstimos e a falta de fontes suficientes de recursos financeiros levam à liquidez inadequada, reduzindo a confiança que o público deposita na cooperativa.

Logaritmo do total de ativos é um indicador utilizado para verificar os impactos do tamanho da instituição na capacidade financeira desta. Os resultados encontrados mostraram que o logaritmo do total de ativos da instituição está diretamente relacionado com a condição de muito baixo risco de liquidez, dado que o aumento marginal desse indicador, na probabilidade de muito baixo risco, foi de $+0,21$, no período analisado. Isso significa que os ganhos pela economia de escala e escopo, provenientes de instituições maiores, são determinantes de boa situação financeira, conforme afirmado por Gaver e Pottier (2005). O impacto nas probabilidades de médio e alto risco de liquidez permaneceu constante, com pequeno valor de $-0,05$. 
Em resumo, aumento nos indicadores utilização de capital de terceiros e provisionamento reduz a probabilidade marginal de as cooperativas analisadas estarem com muito baixo risco de liquidez, aumentando a probabilidade marginal das demais alternativas. Já valores mais elevados dos indicadores depósito total/operações de crédito e logaritmo do total de ativos aumentam a probabilidade de essas cooperativas possuírem boas condições de liquidez, reduzindo os níveis de risco.

A Tabela 6 apresenta a situação real e estimada da classificação das observações nas diferentes faixas de risco consideradas. Verifica-se que os maiores percentuais de acerto das classificações ocorreram para as faixas de muito baixo e muito alto risco de liquidez, sendo de 91,02 e 52,47\%, respectivamente.

\section{Tabela 6: Resultados da Classificação do Modelo da Situação Estimada e Real das Cooperativas de Economia e Crédito Mútuo no Estado de Minas Gerais, no Período de Fevereiro de 2003 a Maio de 2005}

\begin{tabular}{cccc}
\hline $\begin{array}{c}\text { Faixa de risco de } \\
\text { liquidez }\end{array}$ & $\begin{array}{c}\text { Situação estimada da } \\
\text { cooperativa }\end{array}$ & $\begin{array}{c}\text { Situação real da } \\
\text { cooperativa }\end{array}$ & $\begin{array}{c}\text { Percentual de } \\
\text { acerto }\end{array}$ \\
\hline Muito baixo risco & 649 & 713 & $91,02 \%$ \\
Baixo risco & 139 & 325 & $42,77 \%$ \\
Médio risco & 113 & 294 & $38,44 \%$ \\
Alto risco & 163 & 337 & $48,37 \%$ \\
Muito alto risco & 138 & 263 & $52,47 \%$ \\
Total & 1202 & 1932 & $62,22 \%$ \\
\hline
\end{tabular}

Fonte: resultados da pesquisa

Apesar de as faixas de risco intermediárias terem apresentado menor percentual de acerto, a maior frequiência das observações, em todos os casos, incidiu em suas respectivas faixas. É relevante mencionar que trabalhar com número maior de categorias leva naturalmente à maior dispersão dos valores, aumentando os erros de classificação. A proporção total das observações classificadas corretamente foi de $62,22 \%$.

\section{Conclusões e Sugestões}

Para que as cooperativas de economia e crédito mútuo analisadas reduzam os riscos de liquidez, torna-se necessário que especial atenção seja dada ao indicador utilização de capital de terceiros que, em termos de magnitude do efeito marginal, foi o que teve maior influência no risco de liquidez. Esse resultado indica que as cooperativas de crédito analisadas devem buscar meios para aumentar os níveis 
de capitalização de seu quadro social, a fim de manter o equilíbrio entre as duas fontes de recursos da instituição: capital próprio e de terceiros, e garantir maior autonomia financeira.

Pelos resultados encontrados também foi possível constatar que, para que as cooperativas de crédito analisadas mantivessem níveis mais baixos de risco de liquidez, deveriam ter critérios bem definidos na concessão de crédito, pois o aumento das provisões para crédito de liquidação duvidosa, constituída pelos atrasos e inadimplência dos cooperados, apresentou impacto negativo na liquidez dessas cooperativas.

Valores mais elevados dos indicadores depósitos totais/operações de crédito e logaritmo do total de ativos contribuem para manter as cooperativas em níveis mais baixos de risco de liquidez. O tamanho da instituição, representado pelo logaritmo do total de ativos, apesar de significativo em todas as faixas de risco de liquidez consideradas, só exerce influência mais expressiva nas cooperativas que estão com muito baixo risco, o que mostra que este indicador, dentre os analisados, foi o que teve menor relevância para a condição de liquidez.

Pela análise dos dados, foi possível verificar que a maior parte das cooperativas de economia e crédito mútuo de Minas Gerais não está em risco de liquidez, dado que o maior número de observações se concentrou na faixa de muito baixo risco. Esse resultado é decorrente, principalmente, da adequação das cooperativas de economia e crédito mútuo mineiras às exigências das instituições normatizadoras e supervisoras, em relação ao controle do risco de liquidez.

A limitação principal do modelo refere-se ao fato de não ter sido possível calcular indicadores de curto e longo prazo, já que os dados fornecidos estão de acordo com o elenco de contas do Plano Contábil das Instituições Financeiras do Sistema Financeiro Nacional [COSIF], não tendo em sua estrutura a separação entre ativo circulante e realizável a longo prazo e passivo circulante e exigível a longo prazo. Com vistas em sanar essa deficiência, foram consideradas as relações entre as contas disponibilidades e os depósitos à vista.

Apesar de limitado, este estudo buscou contribuir para maior entendimento dos riscos de liquidez nas cooperativas de crédito, identificando aspectos específicos que influenciam a liquidez das cooperativas de economia e crédito mútuo em Minas Gerais.

Para trabalhos futuros, sugere-se o uso de outras metodologias que permitam mitigar e controlar o risco de liquidez, pois a estimação das variáveis que influenciam o risco de liquidez, por si só, não é suficiente. Assim, deve ser associada a análise dos efeitos da diversificação da carteira de ativos, bem como agregadas modelagens como Asset-Liability Model [ALM], que permitam o 
acompanhamento dinâmico dos ativos e passivos da instituição, visando minimizar o risco de liquidez. Podem ser também usadas metodologias para definir o capital mínimo diário que deve estar disponível ou que possa ser rapidamente convertido em dinheiro, para cobrir possíveis perdas ou necessidades mais imediatas. A literatura já oferece instrumentos para o cálculo do capital mínimo exigido, que constituem ferramenta para administração da liquidez.

Sugere-se também, em trabalhos posteriores, que sejam identificadas outras variáveis que venham a impactar a probabilidade de as cooperativas de crédito estarem em risco de liquidez, já que este estudo não utilizou todos os indicadores existentes na análise financeira. Além disso, podem ser realizados estudos com base em informações provenientes de outros estados brasileiros. Assim, poderia ser verificado se as diferenças entre os Estados modificam os determinantes do risco de liquidez. Outros estudos sobre o tema também permitiriam melhor embasamento na definição das políticas de liquidez pelos órgãos supervisores e normatizadores das cooperativas de crédito.

\section{Artigo recebido em 04.02.2006. Aprovado em 11.10.2006.}

\section{Notas}

${ }^{1}$ Passivo Real $=$ Passivo Total - Relações Interfinanceiras - Relações Interdependências .

${ }^{2}$ Captação Total $=$ Passivo Real - Patrimônio Líquido - Diversos.

${ }^{3}$ Aplicação Total $=$ Ativo Real - Ativo Permanente - Diversos; sendo Ativo Real = Ativo Total Relações Interfinanceiras - Relações Interdependências.

${ }^{4}$ Como foram avaliadas 69 cooperativas em 28 meses, obtiveram-se 1.932 observações.

\section{RefERÊnCIAS BibliográficAs}

Aspachs, O.,

Nier, E., \&

Tiesset, M. (2005).

Liquidity, banking regulation and the macroeconomy: evidence on bank liquidity holdings from a panel of UK-resident bank. Recuperado em 2 julho, 2005, de http://econ.lse.ac.uk/ c our s e s / e c $501 / \mathrm{G} / \mathrm{m} \mathrm{m} /$ mm_aspachs.pdf
Assaf, A., Neto (2003).

Estrutura e análise de balanços: um enfoque econômico-financeiro (5a ed.). São Paulo: Atlas.

Banco de Portugal. (2004).

Relatório de estabilidade financeira. Recuperado em 18 julho, 2005, de http://www.bportugal.pt/publish/ref/ anteriores/ref_04_p.pdf 
Bank for International Settlements. (2004, June).

International convergence of capital measurement and capital standards. Recuperado em 20 junho, 2005, de ht t p : / / ww w. bis.org/publ/ bcbs107a.pdf

Bressan, V. G. F. (2002).

Análise de insolvência das cooperativas de crédito rural do estado de Minas Gerais. Dissertação de mestrado. Universidade Federal de Viçosa, Viçosa, MG, Brasil.

Cragg, J. G., \&

Uhler, R. S. (1970).

The demand for automobiles. Canadian Journal of Economics/ Revue canadienne d'Economique, 3(3), 386-406.

Draper, N. R., \&

Smith, H. (1966).

Applied regression analysis. New York: John Wiley \& Sons.

Duarte, A. M., Jr. (2003).

A importância do gerenciamento de riscos corporativos em bancos. In A. M. Duarte Jr. \& G. Varga (Orgs.). Gestão de riscos no Brasil (pp. 3-12). Rio de Janeiro: Financial Consultoria.

Durán, A.,

Matarrita, R., \&

Bronn, D. (2004).

Calificación externa de entidades financieras: su relevancia para CACs en América Latina y lãs implicaciones de Basilea II. San José, C. R.: Confederación Alemana de Cooperativas (DGRV).
Federação Brasileira de Bancos. (2005).

Noções de gestão do risco de liquidez e políticas de contingência. Recuperado em 9 julho, 2005, de http:/ /www.febraban.org.br/Arquivo/ Destaques/Gestao-de-Risco-deMercado.pdf

Gaver, J. J., \&

Pottier, S. W. (2005).

The role of holding company financial information in the insurer-rating process: evidence from the propertyliability industry. Journal of Risk and Insurance, 72(1), 77-103.

Greene, W. (2003).

Econometric analysis (5th ed.). New Jersey: Prentice Hall.

Kaplan, R. S., \& Urwitz, G. (1979).

Statistical models of bond ratings: a methodological inquiry. Journal of Business, 52(2), 231-261.

Lawrence, E. C., \&

Arshadi, N. (1995).

A multinomial logit analysis of problem loan resolution choices in banking. Journal of Money, Credit, and Banking, 27(1), 202-216.

Maddala, G. S. (1986).

Limited-dependent and qualitative variables in econometrics. New York: Cambridge Universit.

Madura, J. (2003).

Financial markets and institutions (6th ed.). Florida: Thompson. 
Matias, A. B., \&

Siqueira, J. O. (1996).

Risco bancário: modelo de previsão de insolvência de bancos no Brasil. Revista de Administração, 31(2), 19-28.

Palia, D., \&

Porter, R. (2003).

Contemporary issues in regulatory risk management of commercial banks. Financial Markets, Institutions \& Instruments, 12(4), 223-256.

Richardson, D. C. (2002).

Pearls monitoring system. Recuperado em 9 julho, 2005, de https:// www.woccu.org/functions/ view_document.php?id=Monograph_4

Saunders, A. (2000).

Administração de instituições financeiras (A. Z. Sanvicente, Trad.). São Paulo: Atlas. (Obra original publicada em 1994).
Schimit, P., \&

Strauss, R. P. (1975).

The predictions of occupation using multiple logit models. International Economic Review, 16(2), 471-486.

Theil, H. (1969).

A multinomial extension of the linear logit model. International Economic Review, 10(3), 251-259.

World Council of Credit Unions. (2002, December).

Development best practices in credit union supervision: regulatory standards. Recuperado em 9 março, 2005, de http://www.woccu.org/ bestpractices/legreg/regupdate

Young, R. D. (2003).

De novo bank exit. Journal of Money, Credit, and Banking, 35(5), 711-728. 
